\title{
Randomized trial of transcranial alternating current stimulation for treatment of auditory hallucinations in schizophrenia ${ }^{2}$
}

\author{
Juliann M. Mellin ${ }^{\mathrm{a}}$, Sankaraleengam Alagapan ${ }^{\mathrm{a}, \mathrm{f}}$, Caroline Lustenberger ${ }^{\mathrm{a}}$, \\ Courtney E. Lugo ${ }^{\mathrm{a}}$, Morgan L. Alexander ${ }^{\mathrm{a}, \mathrm{f}}$, John H. Gilmore ${ }^{\mathrm{a}}$, L. Fredrik Jarskog ${ }^{\mathrm{a}, 1}$, \\ Flavio Fröhlich ${ }^{\mathrm{a}, \mathrm{b}, \mathrm{c}, \mathrm{d}, \mathrm{e}, \mathrm{f}, *, 1}$ \\ a Department of Psychiatry, University of North Carolina at Chapel Hill, Chapel Hill, NC 27599, United States \\ ${ }^{\mathrm{b}}$ Department of Neurology, University of North Carolina at Chapel Hill, Chapel Hill, NC 27599, United States \\ ${ }^{\mathrm{c}}$ Department of Biomedical Engineering, University of North Carolina at Chapel Hill, Chapel Hill, NC 27599, United States \\ d Department of Cell Biology and Physiology, University of North Carolina at Chapel Hill, Chapel Hill, NC 27599, United States \\ e Neuroscience Center, University of North Carolina at Chapel Hill, Chapel Hill, NC 27599, United States \\ ${ }^{\mathrm{f}}$ Carolina Center for Neurostimulation, University of North Carolina at Chapel Hill, Chapel Hill, NC 27599, United States
}

\section{A R T I C L E I N F O}

\section{Article history:}

Received 19 October 2017

Received in revised form 18 January 2018

Accepted 23 January 2018

Available online 11 March 2018

\section{Keywords:}

Auditory hallucinations

Transcranial alternating current stimulation Schizophrenia

\begin{abstract}
A B S T R A C T
Background: Approximately $30 \%$ of patients with schizophrenia experience auditory hallucinations that are refractory to antipsychotic medications. Here, we evaluated the feasibility and efficacy of transcranial alternating current stimulation (tACS) that we hypothesized would improve auditory hallucination symptoms by enhancing synchronization between the frontal and temporo-parietal areas of the left hemisphere.

Method: 22 participants were randomized to one of three arms and received twice daily, 20 min sessions of sham, $10 \mathrm{~Hz} 2 \mathrm{~mA}$ peak-to-peak tACS, or $2 \mathrm{~mA}$ tDCS over the course of 5 consecutive days. Symptom improvement was assessed using the Auditory Hallucination Rating Scale (AHRS) as the primary outcome measure. The Positive and Negative Syndrome Scale (PANSS) and the Brief Assessment of Cognition in Schizophrenia (BACS) were secondary outcomes.

Results: Primary and secondary behavioral outcomes were not significantly different between the three arms. However, effect size analyses show that tACS had the greatest effect based on the auditory hallucinations scale for the week of stimulation (1.31 for tACS; 1.06 and 0.17 , for sham and tDCS, respectively). Effect size analysis for the secondary outcomes revealed heterogeneous results across measures and stimulation conditions.

Conclusions: To our knowledge, this is the first clinical trial of tACS for the treatment of symptoms of a psychiatric condition. Further studies with larger sample sizes are needed to better understand the effect of tACS on auditory hallucinations.
\end{abstract}

(ㄷ) 2018 Elsevier Masson SAS. All rights reserved.

\section{Introduction}

Approximately $30 \%$ of patients diagnosed with schizophrenia experience auditory hallucinations $(\mathrm{AH})$ that are resistant to treatment with antipsychotic medication and are associated with a significant decrease in the quality of life [1]. Non-invasive brain stimulation may provide a viable treatment option for this patient population. For example, transcranial magnetic stimulation (TMS)

\footnotetext{
its STILL 2 Study.

* Corresponding author at: 115 Mason Farm Rd., NRB 4109F, Chapel Hill, NC 27599, United States.

E-mail address: flavio_frohlich@med.unc.edu (F. Fröhlich).

1 Authors contributed equally.
}

has reduced $\mathrm{AH}$ in some, but not all, clinical trials [2]. The reason for this heterogeneity in outcomes remains unknown. In addition, TMS is expensive and needs to be performed in the clinic. In contrast, transcranial current stimulation applies a weak electric current to the scalp and represents a potentially attractive alternative due to the low-cost and portability of the technology [3]. Transcranial direct current stimulation (tDCS) significantly reduced $\mathrm{AH}$ symptoms measured by the Auditory Hallucination Rating Scale (AHRS) in a double-blind, sham controlled study [4]. In this study the anode (assumed to increase neural activity) and the cathode (assumed to decrease neural activity) were placed over the dorso-lateral prefrontal cortex (dl-PFC) to target hypoactivity and the temporo-parietal junction (TPJ) to target hyperactivity, respectively. However, in a recent study from our group with a 
similar design, active tDCS did not separate from sham on the AHRS score, due at least in part to a substantial placebo response to sham stimulation [5]. The reasons for this discrepancy are uncertain but it is notable that the outcomes of these two studies differ primarily in the magnitude of the placebo response. Other studies examining the efficacy of tDCS for the treatment of psychiatric disorders have found mixed results [6,7].

Here, we pursued a novel approach and asked if targeting aberrant temporal organization of brain activity can modulate medication-refractory $\mathrm{AH}$ in schizophrenia patients. This approach was motivated by previous magnetoencephalography (MEG) and electroencephalography (EEG) studies that have noted changes in cortical oscillation patterns and functional connectivity specifically during AH [8-13]. Transcranial alternating current stimulation (tACS) employs a weak electric current for non-invasive brain stimulation similar to tDCS. However, the stimulation current assumes a sine-wave waveform to target brain oscillations in a frequency-specific manner that engages and enhances naturally occurring cortical oscillations at the applied frequency [14-16]. It is hypothesized that alpha oscillations $(8-12 \mathrm{~Hz})$ are generated by

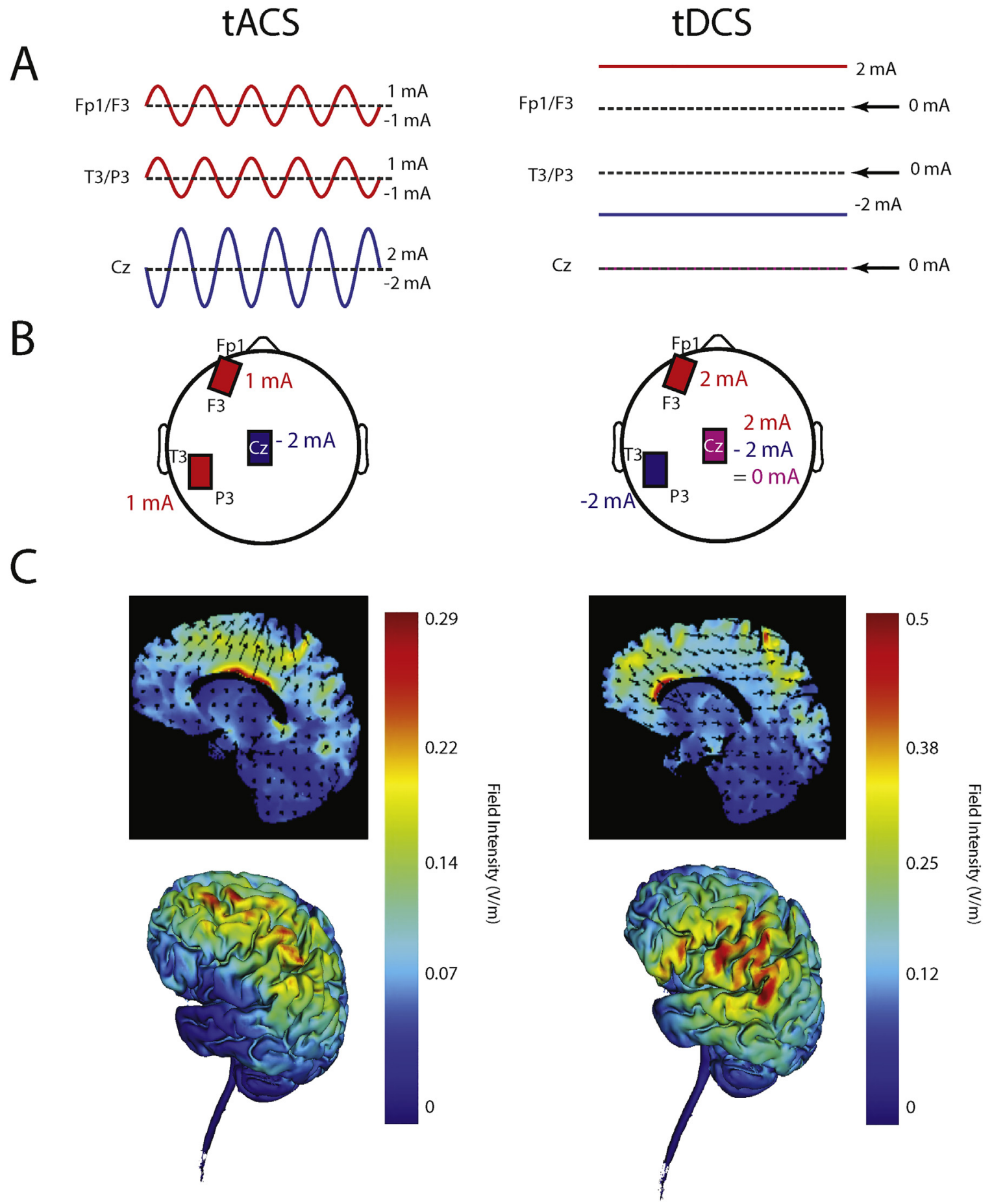

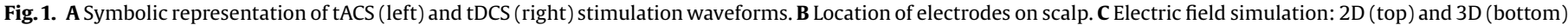

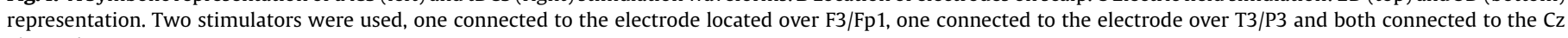
electrode. 
thalamo-cortical and intra-cortical circuits $[17,18]$ making this frequency band susceptible to cortical brain stimulation. For example, a study conducted by Herrmann et al. [19] successfully demonstrated the enhancement of alpha band oscillations using tACS and simultaneous EEG in healthy human participants.

In this study, tACS was used to target alpha oscillations, given the deficits in resting state alpha band power in patients with chronic schizophrenia and first episode psychosis [20,21]. To our knowledge, this represents the first study of tACS in psychiatric patients. This was a double-blind, sham controlled pilot study that compared tDCS, tACS, and sham stimulation in an acrossparticipant design in patients with schizophrenia and persistent $\mathrm{AH}$. Due to the novelty of tACS in schizophrenia, we performed this pilot feasibility study for which we formulated outcomes based on raw effect sizes. We hypothesized that tACS outperforms both tDCS and sham stimulation in terms of reduction of AHRS scores.

\section{Methods}

\subsection{Participants}

This study was conducted at the University of North Carolina at Chapel Hill (ClinicalTrials.gov NCT02360228) and was approved by the UNC Chapel Hill Institutional Review Board. Participants were recruited from local clinics both affiliated and unaffiliated to the university.

\subsubsection{Inclusion criteria}

Participants were diagnosed with schizophrenia or schizoaffective disorder (confirmed by the Structured Clinical Interview for DSM-IV Axis I Disorders); experienced at least three AH per week (as determined by the frequency item in the AHRS); clinical stability as demonstrated by no hospitalizations for the past 3 months; stable dosing of antipsychotic medications (no changes in medication or doses for 1 month prior to enrollment); verified by chart review and/or discussion with the treating clinician to have treatment-persistent $\mathrm{AH}$, defined as having ongoing $\mathrm{AH}$ during trials of at least 2 antipsychotic agents of adequate dose and duration; stable $\mathrm{AH}$ as demonstrated by having less than or equal to $20 \%$ change in AHRS scores across a 2 week interval during the screening period; ability to provide written informed consent.

\subsubsection{Exclusion criteria}

No concurrent anticonvulsant medications or daily treatment with benzodiazepines (limited as-needed use that was discontinued more than $48 \mathrm{~h}$ prior to a study session was allowed); no DSM-IV diagnosis of alcohol or substance abuse within the past month or DSM-IV diagnosis of alcohol or substance dependence within the past 6 months; no history of significant head injury or traumatic brain injury, prior brain surgery or any brain devices/ implants, history of seizures, unstable medical illness, or pregnancy.

This study used a Data Safety Monitoring Board (DSMB), through the North Carolina Translational \& Clinical Sciences Institute to ensure participant safety. Bi-annual reviews of blinded data and adverse events were submitted to the DSMB.

\subsection{Study design}

This study was a double blind, randomized, sham controlled pilot clinical trial, with three study arms ( $10 \mathrm{~Hz} 2 \mathrm{~mA}$ tACS, $2 \mathrm{~mA}$ tDCS, sham $10 \mathrm{~Hz}$, Fig. 1A). The CONSORT diagram is included in the Supplementary materials. Participants were assigned to a code in chronological order based on the date of enrollment. Randomization was blocked such that all three groups had 8 participants. All authors and members of the research team were unaware of the group assignments until completion of the entire study. To administer stimulation in a double-blind fashion, we developed a custom built Matlab (Mathworks, Natick, MA) interface that controlled two Neuroconn DC Plus stimulators (Neuroconn Ltd., Ilmenau, Germany) via the "remote in" feature. This setup provided stimulation linked to the study code and recorded the applied waveform for subsequent verification by a group member not associated with this study.

\subsection{Electrode montage}

All three study arms used the same electrode montage to ensure blinding of the research personnel to the stimulation condition. Three electrodes with ten20 paste (Bio-Medical Instruments, Clinton Township, Michigan) were applied to the scalp. One $5 \times 5 \mathrm{~cm}$ electrode was placed between F3 and Fp1 (left dl-PFC) and one $5 \times 5 \mathrm{~cm}$ electrode was placed between T3 and P3 (left TPJ). A third "return/reference" electrode $(5 \times 7 \mathrm{~cm})$ was placed over Cz. The resulting electric field distribution is shown in Fig. 1C. These figures were created using the Soterix Medical HD-Targets ${ }^{\mathrm{TM}}$ software (Soterix, New York, NY). After specifying the brain region and stimulation electrode location, the resulting simulation depicted the current flow through the head. The location of the stimulation electrodes was determined using the $10-20$ placement system. The choice of location for the stimulation electrodes was motivated by previous tDCS studies for auditory hallucinations in schizophrenia [4,5]. In order to maintain the double blind nature of the study, the location of the tACS electrodes had to necessarily be the same. The stimulation amplitudes for the tACS condition were chosen in a way that the peak amplitude at the third electrode at $\mathrm{Cz}$ never exceeded $2 \mathrm{~mA}$.

\subsection{Stimulation paradigms}

Each participant completed twice-daily $20 \mathrm{~min}$ stimulation sessions, separated by $3 \mathrm{~h}$, over 5 consecutive days. The $10 \mathrm{~Hz}$ tACS stimulation waveform was a sine-wave with a peak-to-peak amplitude of $2 \mathrm{~mA}$. Both stimulators delivered a $2 \mathrm{~mA}$ peak-topeak amplitude current between the frontal site and $\mathrm{Cz}$ and between the temporo-parietal site and $\mathrm{Cz}$, respectively. For tDCS, the stimulation paradigm was $+2 \mathrm{~mA}$ at the frontal site (F3/Fp1) and $-2 \mathrm{~mA}$ at the temporo-parietal site (T3/P3). Sham stimulation included $10 \mathrm{~s}$ of ramp-in to $20 \mathrm{~s}$ of $10 \mathrm{~Hz}$ tACS, with a ramp-out of $10 \mathrm{~s}$ for a total of $40 \mathrm{~s}$ of stimulation. Ramping up and down the stimulation amplitude is a common approach for transcranial current stimulation to reduce skin sensation at stimulation onset. All three conditions used this procedure. The brief stimulation delivered as part of the sham stimulation is unlikely to be biologically active, since duration of tACS appears to be an important variable in terms of the modulation of brain activity after discontinuation of stimulation. Recently, brief periods of tACS were shown to be ineffective in modulating alpha oscillations in healthy human participants [22].

During stimulation, all participants were kept in the same relaxed state. Each participant was seated comfortably upright with their eyes open and asked to focus on the ReefScapes video (Undersea Productions, Queensland, Australia) directly in front of them. This video also served the purpose of helping to disguise the phosphenes induced by tACS.

\subsection{Assessment of side effects}

We administered an adverse effects stimulation questionnaire at the end of each 20 min stimulation session. This assessment was a Likert Scale and measured patient-reported headache, neck pain, scalp pain, tingling, itching, ringing/buzzing noise, burning 
sensation, local redness, sleepiness, trouble concentrating, improved mood, worsening of mood, dizziness, and flickering lights on a scale from 1 (absent) to 4 (severe). After the final stimulation session, participants were asked whether they thought they had received stimulation over the past week, and whether they thought their symptoms $(\mathrm{AH})$ had improved.

\subsection{Screening procedures}

At the initial session, data was collected for each participant regarding demographics, handedness, their belief in the treatment (to understand susceptibility to placebo effect), and current medications which was verified with medical records or treatment providers. All available information from participants, medical records and providers was used to assess whether the AH met study criteria for medication-refractory. All assessments were administered by a researcher blind to the group assignment.

\subsection{Analysis}

For analysis, custom written scripts in $R$ ( $R$ Foundation for Statistical Computing, Vienna, Austria) and SPSS software version 24.0 (IBM, Armonk, NY) were used. Libraries used in R included lme4 [23] and pbkrtest [24]. Differences in demographics and characteristics of the three study arms and the severity of adverse effects were assessed with a one-way ANOVA. Pearson's Correlation was used to assess possible susceptibility to placebo response using the Hunter Beliefs About Treatment Questionnaire, (used with the permission of the UCLA Laboratory of Brain, Behavior and Pharmacology, ${ }^{\odot} 2005,2017$ UC Regents). Pearson's Correlation was also used to examine correlation between age of participant and amount of symptom improvement. We used a linear mixed model analysis with fixed factors of "session" (baseline at day 1 of stimulation, day 5 of stimulation, 1 week follow up, and 1 month follow up) and "condition" (10 Hz tACS, tDCS, active sham $10 \mathrm{~Hz}$ ), with random factor "participant" to account for repeat measures within participants. The interaction between "session" and "condition" is defined as the effects of "session" on "condition". Kenward-Roger approximations were used to calculate $P$-values and perform $F$-tests for each factor and their interaction in the mixed model. Post-hoc analyses included paired $t$-tests to compare the four sessions, with a Bonferroni correction to account for multiple comparisons.

\subsection{Outcome measures}

The primary outcome measure was defined as the change in $\mathrm{AH}$ severity measured by the Auditory Hallucination Rating Scale (AHRS) from baseline (day 1 of stimulation) to day 5 of stimulation. The AHRS was administered before the 1 st stimulation session on day 1 of stimulation and after the 10th stimulation on day 5 of stimulation. The AHRS was also administered at the one-week and the one-month follow up. We also included changes in the oscillatory structure of the resting state EEG data which was collected at 4 time points throughout the study (day 1, day 5, one week follow up and one month follow up). The EEG data will be reported in a separate manuscript.

Secondary outcomes included change in overall symptoms as measured by the Positive and Negative Syndrome Scale (PANSS) and changes in cognitive function as assessed by the Brief Assessment of Cognition in Schizophrenia (BACS) from baseline (day 1 of stimulation) to day 5 of stimulation. Both the PANSS and the BACS were administered before the 1 st session of stimulation, after the 10th session of stimulation, and the one month follow up.

Data was also collected to determine whether participants believed their symptoms $(\mathrm{AH})$ had improved after the 5 days of stimulation with a self-rating questionnaire. Participants were asked at day 5 of stimulation, the one week and one month follow up. During the stimulation week, questionnaires were administered immediately after stimulation, typically with a brief delay of less than $10 \mathrm{~min}$ to give the participant the chance to rinse out electrode paste from their hair.

\section{Results}

\subsection{Study sample}

Twenty-five clinically stable participants with a diagnosis of schizophrenia or schizoaffective disorder were randomized to one of three treatment arms (tACS, tDCS, or active sham). One participant randomized to the tACS group withdrew due to unrelated health concerns (instability of diabetes related symptoms not well controlled by the medication regimen during their participation in the study), a total of 24 participants completed the study (schizophrenia: 15, schizoaffective disorder: 7; 15 men, 7 women). Two participant datasets were not included in analysis due to instability of AH symptoms at baseline and non-adherence to antipsychotic medication during study participation that was unknown to the study team at the time of stimulation. In this paper, we present the analysis of the remaining 22 participants.

\subsection{Safety and tolerability}

Participants in all treatment arms tolerated stimulation well (Table 1 ). Mild tingling, itching and burning were reported by some participants. Some participants in both tACS and sham treatment groups reported the appearance of flashing lights, likely related to phosphenes that result from retinal stimulation or, potentially, stimulation of visual cortex by tACS. Group-averaged side-effect scores did not exceed a value of 2 (on a scale from 1 to 4 ) and there were no statistically significant differences between groups for the averaged total score $(p=.31)$. There were no significant adverse events reported throughout the entirety of the study. All participants who completed the study were able to sit through all 10 stimulation sessions during the treatment week. Participants who decided not to continue with their participation in the study withdrew for reasons unrelated to the stimulation itself, as reported to study personnel.

Table 1

Stimulation Side Effect Scores.

\begin{tabular}{|c|c|c|c|c|c|c|c|}
\hline \multirow[t]{2}{*}{ Side Effect } & \multicolumn{2}{|l|}{ tACS } & \multicolumn{2}{|l|}{ tDCS } & \multicolumn{2}{|l|}{ Sham } & \multirow[b]{2}{*}{ p-value } \\
\hline & Mean & SD & Mean & SD & Mean & SD & \\
\hline Headache & 1.2 & 0.39 & 1.0 & 0 & 1.3 & 0.63 & .30 \\
\hline Neck pain & 1.0 & 0 & 1.0 & 0.17 & 1.2 & 0.48 & .07 \\
\hline Scalp pain & 1.5 & 0.57 & 1.4 & 0.72 & 1.5 & 0.85 & .94 \\
\hline Tingling & 1.9 & 0.44 & 2.1 & 0.99 & 2.0 & 0.98 & .84 \\
\hline Itching & 1.6 & 0.65 & 2.1 & 0.97 & 1.4 & 0.67 & .18 \\
\hline Ringing/Buzzing noise & 1.0 & 0 & 1.0 & 0.12 & 1.0 & 0.12 & .57 \\
\hline Burning sensation & 1.6 & 0.56 & 2.4 & 0.96 & 2.1 & 1.11 & .17 \\
\hline Local redness & 1.0 & 0 & 1.0 & 0 & 1.0 & 0.12 & .36 \\
\hline Sleepiness & 1.2 & 0.37 & 1.9 & 1.07 & 1.9 & 0.9 & .03 \\
\hline Trouble concentrating & 1.2 & 0.39 & 1.5 & 0.79 & 1.6 & 0.67 & .33 \\
\hline Improved mood & 1.4 & 0.58 & 1.2 & 0.4 & 1.5 & 0.65 & .49 \\
\hline Worsening of mood & 1.0 & 0 & 1.0 & 0 & 1.1 & 0.24 & .17 \\
\hline Dizziness & 1.0 & 0 & 1.1 & 0.44 & 1.3 & 0.54 & .05 \\
\hline Flickering Lights & 1.3 & 0.53 & 1.0 & 0 & 1.5 & 0.85 & .16 \\
\hline
\end{tabular}

tACS: transcranial alternating current stimulation; tDCS: transcranial direct current stimulation.

Values in bold indicate $\mathrm{p}$-values below significance threshold $\mathrm{p}=0.05$. 


\subsection{Auditory Hallucination Rating Scale (AHRS)}

Effect size calculations of baseline to day 5 of stimulation resulted in the largest effect size for tACS (1.31), followed by the sham and the tDCS arms (1.06 and 0.17, respectively, Supplementary Table 1). The tACS group displayed a mean improvement of 15\% (mean: -3.75 points, SD 2.87), the tDCS group displayed a mean improvement of $5 \%$ (mean: -1.14 points SD 6.15 ), and the sham group had a mean improvement of $10 \%$ (mean: -2.29 points SD 2.14) after 5 consecutive days of twice daily stimulation (Fig. 2, Table 2; individual trajectories in Fig. 3). An exploratory F-test with Kenward-Roger approximation analysis was conducted examining factors "session", "condition" and the interaction between "session" and "condition". These factors were compared across time points "baseline", "day 5 of stimulation", "1 week" and " 1 month". The interaction analysis examined whether there was an impact of "session" on "condition" for the AHRS scores. Factor "session" was found to be significant $\left(F_{3,63}=5.05, P<.01\right)$. Analyses conducted with a Bonferroni correction resulted in a difference between "baseline" and "1 month". Factors "condition" and the interaction were not significant (Table 3).

\subsection{Positive and Negative Syndrome Scale (PANSS)}

Effect size calculations of baseline to day 5 of stimulation show the largest effect size for tDCS (1.13), with tACS and sham having a small effect size (0.42 and 0.39 , respectively, Supplementary Table 2). Effect size calculations were also conducted for the PANSS Hallucinations question. Results of this analysis showed that tACS had the largest effect size at baseline to day 5 of stimulation (0.48), while tDCS also had a small effect size (0.30) and sham had no effect size (0.00, Supplementary Table 3$)$. An exploratory analysis of the PANSS total scores with a linear mixed model showed that factor "session" was significant (F-test with Kenward-Roger approximation with time points "baseline", "1 week", and "1 month"; $F_{2,42}=4.95, P=.01$ ). We found no significant effects for the factor "condition" or the interaction for the PANSS total score. No significant effect was found for factors "session", "condition", or the interaction for the positive symptom subscale. No significant effect was found for "condition" or the interaction for the negative symptom subscale. However there was a significant effect found for factor "session" $\left(F_{2,42}=6.87, P=.003\right)$ for the negative symptom subscale. No significant effect was found for the factors "session", "condition", or the interaction for the general psychopathology subscale. No significant effect was found for the factors "session", "condition", or the interaction for the hallucination question in the positive symptom subscale. Results are presented in Table 2.

\subsection{Brief Assessment of Cognition in Schizophrenia (BACS)}

Effect size calculations of baseline to day 5 of stimulation show the largest effect size for tDCS (1.50), with sham having a medium effect size (0.57) and tACS having a small effect size (0.26, Supplementary Table 4). In an exploratory analysis of the BACS total scores with a mixed linear model, we found a significant effect for factor "session" (F-test with Kenward-Roger approximation with the time points "baseline", "1 week", and "1 month"; $\mathrm{F}_{2,42}=4.31, P=.02$ ). No significant effect was found for factor "condition" or the interaction. Results can be seen in Table 2 .

${ }^{*}$ Results from Participant Demographics, Participant Expectation of Outcomes, Self-Rating of Improvement, and Participant Age and AHRS Improvement can be found in Supplementary materials, along with the corresponding tables and figures.

\section{Discussion}

\section{1. $t D C S$ efficacy}

Although several studies have examined tDCS, conclusive results have not emerged as to whether it represents an effective treatment of $\mathrm{AH}$. Studies examining once daily tDCS, either over 5 consecutive days [4] or 3 consecutive weeks (15 total stimulation sessions) [25], did not find significant changes in severity of $\mathrm{AH}$. Studies conducted by Brunelin et al. [4] and Mondino et al. [26] looked at twice daily tDCS for the treatment of AH in patients with schizophrenia, both of which had positive results. In fact, one study found that the improvement in $\mathrm{AH}$ remained apparent through the 3 month follow up [4]. Interestingly, although the treatment

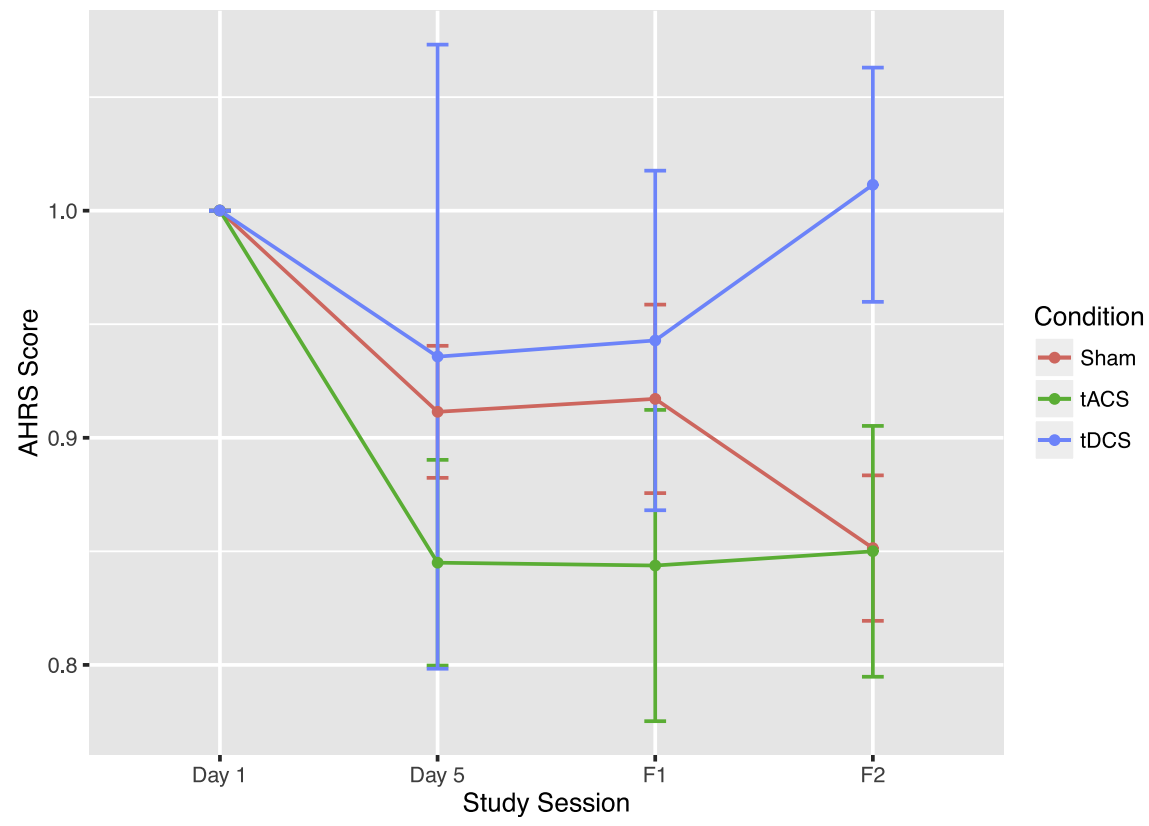

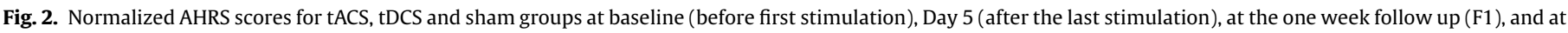
the one month follow up (F2). 
Table 2

AHRS, PANSS and BACS scores.

\begin{tabular}{|c|c|c|c|c|c|c|c|}
\hline \multirow[t]{2}{*}{ Measure } & \multicolumn{2}{|c|}{ tACS $(n=8)$} & \multicolumn{2}{|c|}{$\operatorname{tDCS}(\mathrm{n}=7)$} & \multicolumn{2}{|c|}{ Sham $(n=7)$} & \multirow[b]{2}{*}{ p-value } \\
\hline & Mean & SD & Mean & SD & Mean & SD & \\
\hline Age (years) & 47 & 9.72 & 29.57 & 10.97 & 38.86 & 10.01 & .01 \\
\hline \multicolumn{8}{|l|}{ Baseline Measures } \\
\hline $\begin{array}{l}\text { AHRS Total Score } \\
\text { PANSS }\end{array}$ & 25.88 & 3.8 & 23 & 6.9 & 24.71 & 5.91 & .61 \\
\hline Total Score & 52.75 & 7.74 & 58.86 & 14.66 & 57 & 11.6 & .58 \\
\hline Positive Symptoms & 15.5 & 3.96 & 17.71 & 3.35 & 16.57 & 4.4 & .54 \\
\hline Negative Symptoms & 13.13 & 4.58 & 13.71 & 7.23 & 13.29 & 3.9 & .98 \\
\hline General Psychopathology & 24.13 & 3.72 & 27.43 & 5.8 & 26.71 & 7.95 & .53 \\
\hline Hallucinations & 4.63 & 0.52 & 4.57 & 0.79 & 4.57 & 0.79 & .99 \\
\hline BACS Total Score & 31.21 & 5.68 & 38.26 & 9.01 & 38.93 & 7.17 & .10 \\
\hline \multicolumn{8}{|c|}{ After tACS/tDCS/sham stimulation } \\
\hline $\begin{array}{l}\text { AHRS Total Score } \\
\text { PANSS }\end{array}$ & 22.13 & 5.82 & 21.86 & 9.58 & 22.43 & 5.56 & .99 \\
\hline Total Score & 51.25 & 8.19 & 54.71 & 11.84 & 55.29 & 10.86 & .71 \\
\hline Positive Symptoms & 15.88 & 4.29 & 16.00 & 2.77 & 16.14 & 4.30 & .99 \\
\hline Negative Symptoms & 11.75 & 3.11 & 12.00 & 6.51 & 13.00 & 4.08 & .87 \\
\hline General psychopathology & 23.63 & 3.34 & 26.71 & 5.15 & 26.14 & 6.94 & .49 \\
\hline Hallucinations & 4.38 & 0.52 & 4.14 & 1.68 & 4.57 & 0.79 & .76 \\
\hline BACS Total Score & 31.58 & 5.77 & 42.05 & 10.82 & 41.50 & 10.52 & .07 \\
\hline \multicolumn{8}{|l|}{1 week follow up } \\
\hline AHRS Total Score & 21.88 & 6.22 & 21.43 & 6.55 & 22.29 & 4.19 & .98 \\
\hline \multicolumn{8}{|l|}{1 month follow up } \\
\hline \multicolumn{8}{|l|}{ PANSS } \\
\hline Total Score & 51.13 & 9.95 & 52.86 & 7.47 & 51.57 & 12.20 & .94 \\
\hline Positive Symptoms & 15.63 & 4.17 & 15.86 & 2.27 & 16.71 & 4.82 & .86 \\
\hline Negative Symptoms & 11.50 & 3.30 & 12.29 & 7.11 & 10.57 & 2.23 & .79 \\
\hline General Psychopathology & 24.00 & 6.00 & 24.71 & 3.45 & 24.29 & 6.82 & .97 \\
\hline Hallucinations & 4.25 & 0.46 & 4.57 & 0.98 & 4.43 & 0.79 & .72 \\
\hline BACS Total Score & 29.81 & 5.50 & 39.71 & 9.39 & 40.83 & 9.71 & .03 \\
\hline
\end{tabular}

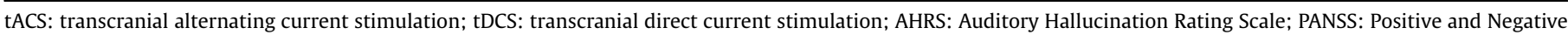
Syndrome Scale; BACS: Brief Assessment of Cognition in Schizophrenia.

Values in bold indicate $\mathrm{p}$-values below significance threshold $\mathrm{p}=0.05$.
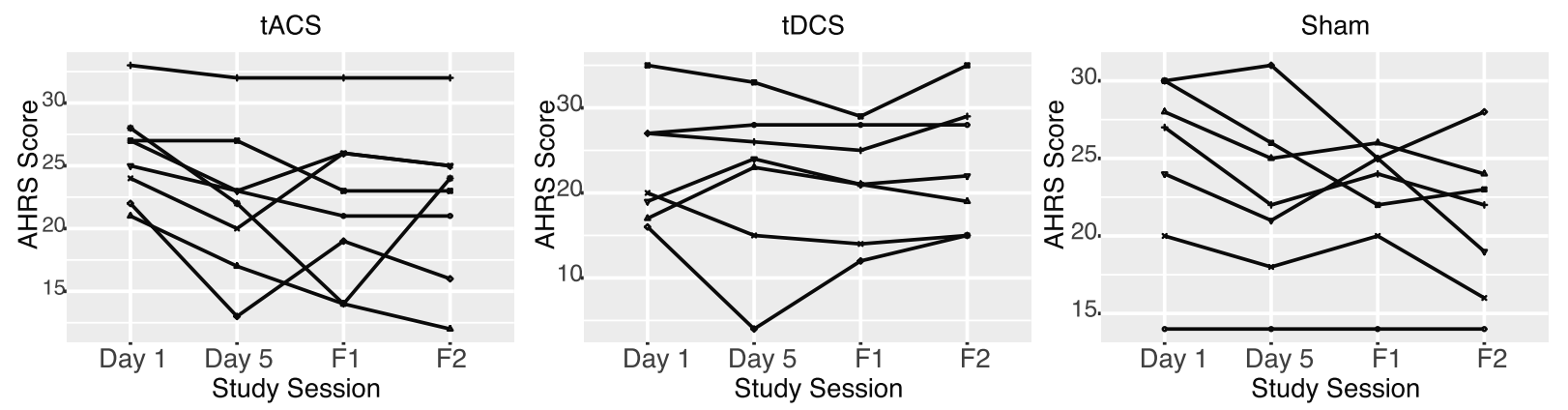

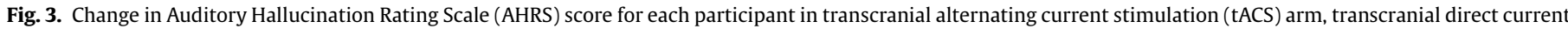

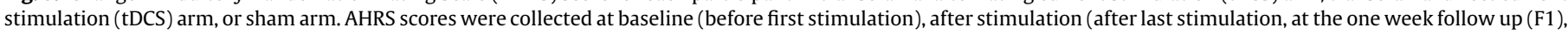
and the one month follow up (F2)).

duration used in our current study mirrored the treatment duration in [4], there were no sustained improvement in $\mathrm{AH}$ past the one week follow up in our study. Although the current trial was smaller, each study was double blinded and randomized, with similar study inclusion/exclusion criteria. It is not clear why these studies differ substantially in response to tDCS in the same population. These inconsistent findings indicate that the benefits of tDCS for persistent $A H$ remain uncertain and will require further study.

\section{2. tACS dosage}

Although the stimulation paradigm mirrored the twice daily treatment in the Brunelin study [4] which found improvement in AH symptoms up to 3 months after the week of stimulation, the present study did not find benefits of tACS past the one week follow up in terms of raw effect sizes when compared to sham stimulation. The tACS and sham arms had similar effect sizes for the difference from "Day 1 " to "1 Week Follow-Up", while the sham arm had the largest effect size for difference from "Day 1" to "1 
Table 3

F-test with Kenward-Roger Approximation Analysis Results.

\begin{tabular}{|c|c|c|c|c|c|c|}
\hline \multirow[t]{2}{*}{ Factor } & \multicolumn{2}{|l|}{ Session } & \multicolumn{2}{|l|}{ Condition } & \multicolumn{2}{|c|}{ Interaction (Session $\times$ Condition) } \\
\hline & F-value & $\mathrm{p}$-value & F-value & p-value & F-value & p-value \\
\hline AHRS Total Score & $\mathrm{F}_{3,63}=5.05$ & .003 & $\mathrm{~F}_{2,19}=0.02$ & .97 & $\mathrm{~F}_{6,57}=1.16$ & .34 \\
\hline \multicolumn{7}{|l|}{ PANSS } \\
\hline Total Score & $\mathrm{F}_{2,42}=4.95$ & .01 & $\mathrm{~F}_{2,19}=0.28$ & .76 & $\mathrm{~F}_{4,38}=0.72$ & .59 \\
\hline Positive Symptoms & $\mathrm{F}_{2,42}=1.02$ & .37 & $\mathrm{~F}_{2,19}=0.13$ & .88 & $\mathrm{~F}_{4,38}=1.73$ & .16 \\
\hline Negative Symptoms & $\mathrm{F}_{2,42}=6.87$ & .003 & $\mathrm{~F}_{2,19}=0.03$ & .97 & $\mathrm{~F}_{4,38}=1.27$ & .30 \\
\hline General Psychopathology & $\mathrm{F}_{2,42}=1.74$ & .19 & $\mathrm{~F}_{2,19}=0.42$ & 66 & $\mathrm{~F}_{4,38}=0.56$ & 69 \\
\hline Hallucinations & $F_{2,42}=1.43$ & .25 & $F_{2,19}=0.04$ & .96 & $\mathrm{~F}_{4,38}=1.01$ & .41 \\
\hline BACS Total Score & $\mathrm{F}_{2,42}=4.31$ & .02 & $\mathrm{~F}_{2,19}=3.44$ & .05 & $\mathrm{~F}_{4,38}=1.40$ & .25 \\
\hline
\end{tabular}

AHRS: Auditory Hallucination Rating Scale; PANSS: Positive and Negative Syndrome Scale; BACS: Brief Assessment of Cognition in Schizophrenia.

Values in bold indicate $\mathrm{p}$-values below significance threshold $\mathrm{p}=0.05$.

Month". It is possible that the duration of treatment may need to be extended past the five day mark in order to sustain symptom improvement. Early TMS studies for depression administered stimulation in short periods for a total of two weeks [27], while it has become common practice for treatments to last in durations of four to six weeks [28]. Future studies may consider increasing the frequency or duration of treatment.

\subsection{Effect sizes}

Due to the small sample size used in this study, statistically significant results were not expected. Raw effect sizes were used to understand the effect that tACS, tDCS and sham had on AH symptoms. As shown, tACS had the largest effect size for the AHRS. However, tDCS had the largest effect sizes for the PANSS and BACS assessments. The divergence of improvement on these different scales may be explained by the multifaceted nature of the disease. Just as not all antipsychotics produce the same improvement in a specific symptom, the different stimulation paradigms may not affect the same symptom clusters. The large effect for the BACS in the tDCS group is intriguing given the emerging literature of anodal tDCS applied to left prefrontal cortex for remediating cognitive deficits in patients with schizophrenia $[7,29]$. We performed an exploratory analysis to understand to what extent this effect on cognition was a result of the significantly different mean ages across the three groups. Across all participants, we found no significant correlation between age and the difference in BACS scores from day 1 to day $5(\mathrm{r}=0.004, \mathrm{n}=22, \mathrm{p}=.78)$. This suggests that the effect of tDCS on cognition is not uniquely an artefact of the uneven age distribution across the three study arms.

\subsection{Blinding}

In contrast to tDCS, tACS can induce the appearance of flashing lights, or phosphenes, that are caused by stimulation of the optical nerve [30]. Several steps were taken in this study to ensure the participants were unable to distinguish whether they had been assigned to the tACS, tDCS or sham group. The sham was designed to mimic the skin sensations of the tACS group in order to blind participants assigned to this group (sometimes referred to as "active"). All participants were asked to sit still with their eyes open while a ReefScapes video was played on a projector screen in front of them which displayed underwater sceneries with tropical fish. The shifting sunlight of the water and the flashing colors of the fish served as a method of disguising the phosphenes induced by tACS and the sham. The blinding of this study was successful, with only one participant believing that they had not received stimulation.

\subsection{Electric field distribution}

We emphasize that the two groups that received active stimulation differed in the waveform of the stimulation used and also in terms of the spatial targeting. In designing this study, we prioritized the full blinding of study participants and researchers to the assigned stimulation condition. In this decision, we have accepted this limitation of the study.

\subsection{Limitations}

There are several limitations to this study that should be addressed. This was an exploratory study with a low number of participants. Due to this small sample size and 3 separate arms, the study was only powered to detect very large effect size changes. Future studies should examine tACS with a fully powered sample size. As this was a randomized treatment study with no blocking for age, the average age of the tACS treatment arm was significantly higher than the tDCS and the sham group. It is possible that the age of the tACS group diminished the effects of the stimulation, as neuroplasticity can decrease with age [31,32]. As a result, our study may underestimate the effect of tACS. Future studies should examine whether a younger population may demonstrate enhanced effects with tACS. The choice of tACS was motivated by targeting long-range functional interactions between the targeted cortical sites. Future analysis of the EEG data and subsequent studies with more targeted neuroimaging to examine changes in structural and functional connectivity will be needed to delineate to what extent clinical improvement is indeed driven by connectivity changes. Lastly, we decided to position the electrodes on the same scalp locations for all groups to allow for successful blinding of the research personnel. As a result, the spatial electric field distribution for the tACS and tDCS group is not exactly identical.

\section{Conclusions}

In this first study to examine the effects of tACS on persistent auditory hallucinations in patients with schizophrenia, the results indicate a difference in symptom response between tACS and tDCS. Further research is needed with a larger sample size and longer treatment duration to better understand the treatment possibilities with tACS and the effects on auditory hallucinations in patients with schizophrenia.

\section{Disclosures of interest}

Flavio Frohlich is the lead inventor of an IP filed by UNC. The clinical studies performed in the Frohlich Lab have received a 
designation as conflict of interest with administrative considerations. Flavio Frohlich is the founder, CSO and majority owner of Pulvinar Neuro LLC. No devices from Pulvinar Neuro LLC were used and Pulvinar Neuro LLC played no role in this study.

\section{Acknowledgements}

The authors thank Kristen Sellers and Zhe Charles Zhou for their help with patient randomization for this study. Research reported in this publication was supported by the National Institute of Mental Health of the National Institutes of Health under Award Number 1R21MH105574-01. The content is solely the responsibility of the authors and does not necessarily represent the official views of the National Institutes of Health. This work was also partially supported by the UNC Department of Psychiatry and UNC School of Medicine. Caroline Lustenberger was supported by the Swiss National Science Foundation, grant P300PA_164693. The project described was supported by the National Center for Advancing Translational Sciences (NCATS), National Institutes of Health, through Grant Award Number UL1TR001111. The content is solely the responsibility of the authors and does not necessarily represent the official views of the $\mathrm{NIH}$.

\section{Appendix A. Supplementary data}

Supplementary data associated with this article can be found, in the online version, at https://doi.org/10.1016/j.eurpsy.2018.01.004.

\section{References}

[1] Shergill SS, Murray RM, McGuire PK. Auditory hallucinations: a review of psychological treatments. Schizophr Res 1998;32(3):137-50.

[2] Cole JC, Green Bernacki C, Helmer A, Pinninti N, O'Reardon JP. Efficacy of transcranial magnetic stimulation (TMS) in the treatment of schizophrenia: a review of the literature to date. Innov Clin Neurosci 2015;12(7-8):12-9.

[3] Philip NS, Nelson BG, Frohlich F, Lim KO, Widge AS, Carpenter LL. Low-intensity transcranial current stimulation in psychiatry. Am J Psychiatry 2017;174 (7):628-39.

[4] Brunelin J, Mondino M, Gassab L, Haesebaert F, Gaha L, Suaud-Chagny MF, et al. Examining transcranial direct-current stimulation (tDCS) as a treatment for hallucinations in schizophrenia. Am J Psychiatry 2012;169(7):719-24.

[5] Frohlich F, Burrello TN, Mellin JM, Cordle AL, Lustenberger CM, Gilmore JH, et al. Exploratory study of once-daily transcranial direct current stimulation (tDCS) as a treatment for auditory hallucinations in schizophrenia. Eur Psychiatry 2016;33:54-60.

[6] Kekic M, Boysen E, Campbell IC, Schmidt U. A systematic review of the clinical efficacy of transcranial direct current stimulation (tDCS) in psychiatric disorders. J Psychiatr Res 2016;74:70-86.

[7] Mervis JE, Capizzi RJ, Boroda E, MacDonald 3rd AW. Transcranial direct current stimulation over the dorsolateral prefrontal cortex in schizophrenia: a quantitative review of cognitive outcomes. Front Hum Neurosci 2017;11:44.

[8] Ropohl A, Sperling W, Elstner S, Tomandl B, Reulbach U, Kaltenhauser M, et al Cortical activity associated with auditory hallucinations. Neuroreport 2004;15 (3):523-6.

[9] Sritharan A, Line P, Sergejew A, Silberstein R, Egan G, Copolov D. EEG coherence measures during auditory hallucinations in schizophrenia. Psychiatry Res 2005;136(2-3):189-200.

[10] Ishii R, Shinosaki K, Ikejiri Y, Ukai S, Yamashita K, Iwase M, et al. Theta rhythm increases in left superior temporal cortex during auditory hallucinations in schizophrenia: a case report. Neuroreport 2000;11(14):3283-7.

[11] Reulbach U, Bleich S, Maihofner C, Kornhuber J, Sperling W. Specific and unspecific auditory hallucinations in patients with schizophrenia: a magnetoencephalographic study. Neuropsychobiology 2007;55(2):89-95.

[12] Angelopoulos E, Koutsoukos E, Maillis A, Papadimitriou GN, Stefanis C. Cortical interactions during the experience of auditory verbal hallucinations. J Neuropsychiatry Clin Neurosci 2011;23(3):287-93.

[13] Lawrie SM, Buechel C, Whalley HC, Frith CD, Friston KJ, Johnstone EC. Reduced frontotemporal functional connectivity in schizophrenia associated with auditory hallucinations. Biol Psychiatry 2002;51(12):1008-11.

[14] Herrmann CS, Rach S, Neuling T, Struber D. Transcranial alternating current stimulation: a review of the underlying mechanisms and modulation of cognitive processes. Front Hum Neurosci 2013;7:279.

[15] Schmidt SL, Iyengar AK, Foulser AA, Boyle MR, Frohlich F. Endogenous cortical oscillations constrain neuromodulation by weak electric fields. Brain Stimul 2014;7(6):878-89.
[16] Vossen A, Gross J, Thut G. Alpha power increase after transcranial alternating current stimulation at alpha frequency (alpha-tACS) reflects plastic changes rather than entrainment. Brain Stimul 2015;8(3):499-508.

[17] Bollimunta A, Mo J, Schroeder CE, Ding M. Neuronal mechanisms and attentional modulation of corticothalamic alpha oscillations. J Neurosci 2011;31(13):4935-43.

[18] Hindriks R, van Putten MJ. Thalamo-cortical mechanisms underlying changes in amplitude and frequency of human alpha oscillations. Neuroimage 2013;70:150-63.

[19] Zaehle T, Rach S, Herrmann CS. Transcranial alternating current stimulation enhances individual alpha activity in human EEG. PLoS One 2010;5(11): e13766.

[20] Goldstein MR, Peterson MJ, Sanguinetti JL, Tononi G, Ferrarelli F. Topographic deficits in alpha-range resting EEG activity and steady state visual evoked responses in schizophrenia. Schizophr Res 2015;168(1-2):145-52.

[21] Sun J, Tang Y, Lim KO, Wang J, Tong S, Li H, et al. Abnormal dynamics of EEG oscillations in schizophrenia patients on multiple time scales. IEEE Trans Biomed Eng 2014;61(6):1756-64.

[22] Strüber D, Rach S, Neuling T, Herrmann CS. On the possible role of stimulation duration for after-effects of transcranial alternating current stimulation. Front Cell Neurosci 2015;9:311.

[23] Bates D, Machler M, Bolker B, Walker S. Fitting linear mix-effects models using lme4. J Stat Softw 2015;67:1-48.

[24] Halekoh U, Hojsgaard S. A kenward-roger approximation and parametric bootstrap methods for tests in linear mixed models-the R package pbkrtest. J Stat Softw 2014;59:1-32.

[25] Fitzgerald PB, McQueen S, Daskalakis ZJ, Hoy KE. A negative pilot study of daily bimodal transcranial direct current stimulation in schizophrenia. Brain Stimul 2014:7(6):813-6.

[26] Mondino M, Jardri R, Suaud-Chagny MF, Saoud M, Poulet E, Brunelin J. Effects of fronto-temporal transcranial direct current stimulation on auditory verbal hallucinations and resting-state functional connectivity of the left temporoparietal junction in patients with schizophrenia. Schizophr Bull 2016;42 (2):318-26.

[27] Daskalakis ZJ, Levinson AJ, Fitzgerald PB. Repetitive transcranial magnetic stimulation for major depressive disorder: a review. Can J Psychiatry 2008;53 (9):555-66

[28] Carpenter LL, Janicak PG, Aaronson ST, Boyadjis T, Brock DG, Cook IA, et al. Transcranial magnetic stimulation (Tms) for major depression: a multisite, naturalistic, observational study of acute treatment outcomes in clinical practice. Depress Anxiety 2012;29(7):587-96.

[29] Hoy KE, Arnold SL, Emonson MR, Daskalakis ZJ, Fitzgerald PB. An investigation into the effects of tDCS dose on cognitive performance over time in patients with schizophrenia. Schizophr Res 2014;155(1-3):96-100.

[30] Kar K, Krekelberg B. Transcranial electrical stimulation over visual cortex evokes phosphenes with a retinal origin. J Neurophysiol 2012;108(8):2173-8.

[31] Spriggs MJ, Cadwallader CJ, Hamm JP, Tippett LJ, Kirk IJ. Age-related alterations in human neocortical plasticity. Brain Res Bull 2017;130:53-9.

[32] Goh JO, Park DC. Neuroplasticity and cognitive aging: the scaffolding theory of aging and cognition. Restor Neurol Neurosci 2009;27(5):391-403.

Juliann M. Mellin is a study coordinator in the Carolina Center for Neurostimulation and Department of Psychiatry at the University of North Carolina Chapel Hill. She received her undergraduate degree in Psychology from the University of North Carolina Greensboro. Her research interests include brain stimulation as a possible treatment for Schizophrenia and Major Depressive Disorder.

Dr. Sankaraleengam Alagapan is a postdoctoral research associate in the Carolina Center for Neurostimulation and Department of Psychiatry at the University of North Carolina Chapel Hill. He received his PhD in Biomedical Engineering from University of Florida. His research interests include brain stimulation, cognition and biological signal processing.

Dr. Caroline Lustenberger is currently a postdoctoral associate at the ETH Zurich in the Mobile Health Systems Lab led by Prof. Walter Karlen. Since 2013, she holds a $\mathrm{PhD}$ in Neuroscience with a focus on sleep and memory from the ETH Zurich (group of Prof. Reto Huber, Children's Hospital Zurich). From 2014 to 2017 she performed a postdoctoral stay at the University of North Caroline at Chapel Hill with Prof. Flavio Frohlich where she gained expertise in advanced brain stimulation approaches. Her research investigates the role of cortical activity during sleep in brain and body health using advanced feedback-controlled brain stimulation techniques.

Courtney E. Lugo is a study coordinator in the Carolina Center for Neurostimulation and Department of Psychiatry at the University of North Carolina at Chapel Hill. She received her Masters in Public Health and her Bachelors of Science in Biology from East Carolina University. Her research areas include Major Depressive Disorder and Schizophrenia. 
Morgan L. Alexander is a study coordinator in the Carolina Center for Neurostimulation and Department of Psychiatry at the University of North Carolina at Chapel Hill. She received her undergraduate degrees in psychology and mathematics from the University of North Carolina at Chapel Hill. She is interested in how novel treatments, such as brain stimulation, can treat patients with severe mental illnesses.

Dr. John H. Gilmore is the Thad and Alice Eure Distinguished Professor and Vice Chair for Research and Scientific Affairs in the Department of Psychiatry at the University of North Carolina at Chapel Hill and directs the UNC Center for Excellence in Community Mental Health. Dr. Gilmore received his BA in Political and Socia Thought from the University of Virginia and his MD from the University of North Carolina at Chapel Hill. Dr. Gilmore's has an active research program focused on brain development and risk for schizophrenia. He pioneered the use of magnetic resonance imaging to study early childhood brain development.
Dr. L. Fredrik Jarskog is Professor of Psychiatry and Research Director of the North Carolina Psychiatric Research Center at the University of North Carolina at Chapel Hill. Dr. Jarskog's research is focused on the pathophysiology and treatment of schizophrenia. He currently receives research funding from the National Institute of Health, several academic/industry collaborations and The Foundation of Hope for Research and Treatment of Mental Illness, Raleigh, NC. Current studies include novel treatments for cognitive deficits in people with schizophrenia, treatmentresistant psychosis, tardive dyskinesia and antipsychotic-associated weight gain.

Dr. Flavio Frohlich received a degree in electrical engineering at ETH Zurich, an International Diploma at the Imperial College in London, and his $\mathrm{PhD}$ in neurobiology at UC San Diego. After a postdoctoral fellowship at Yale University, he joined UNC - Chapel Hill as faculty, where he currently is an associate professor. He directs the Frohlich Lab, which studies brain oscillations in animal models and computer simulations, and the Carolina Center for Neurostimulation, which focuses on research and clinical use of brain stimulation. Dr. Frohlich authored the textbook Network Neuroscience and is the founder and chief scientific officer of Pulvinar Neuro LLC. 